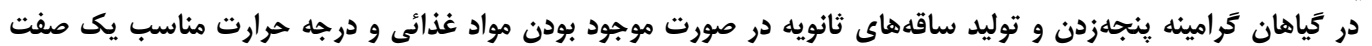

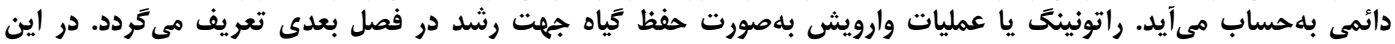

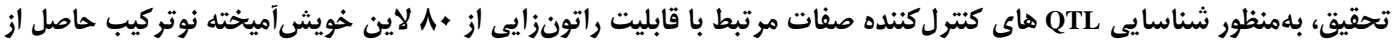

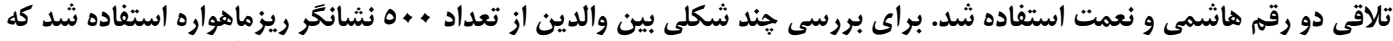

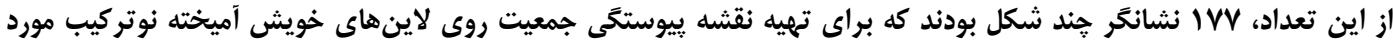

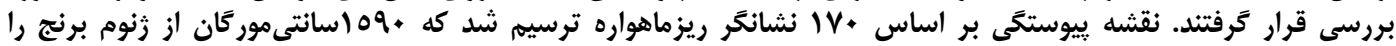

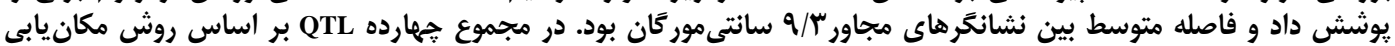

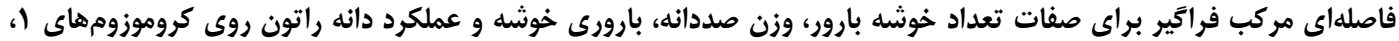

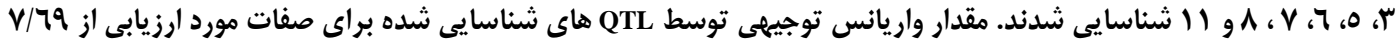

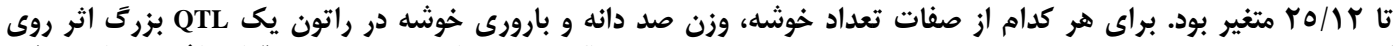

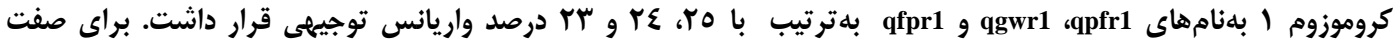

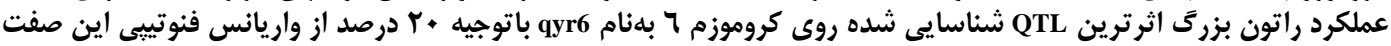

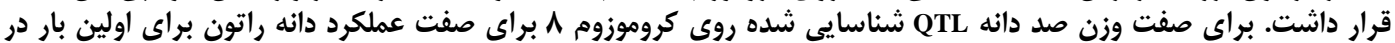
اين تحقيق شناسايى شد.

وازههاى كليدى: برنج، راتونزائى، لاينهاى نوتركيب، نقشهيابى QTL، نشانتر SSR

بيشتر براى توليد راتون در استان را ضرورى مىسازد. برداشت

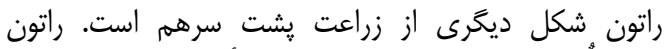

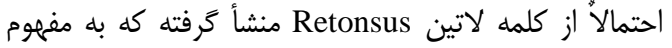
قطع كردن يا درو مىباشد (ج).

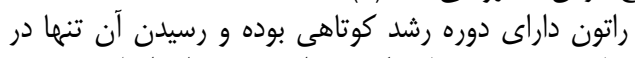

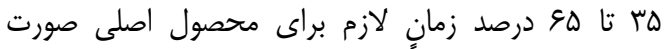

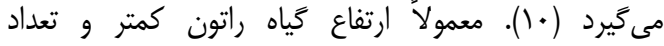

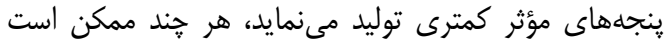

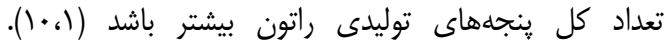

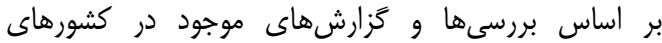

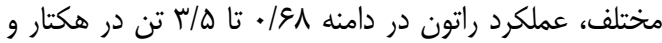

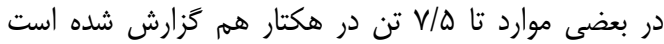

تان و همكاران (IV) با بررسى لاينهاى دابلهايلوئيد

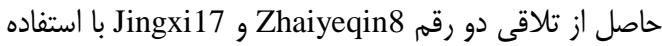

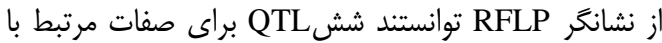

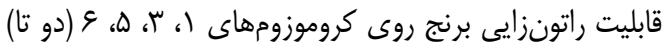

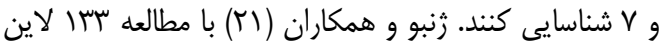

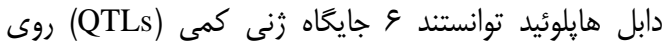

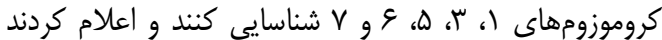

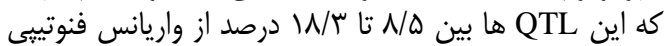

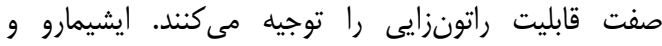

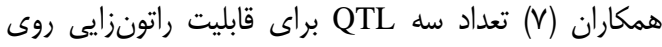

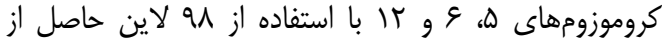

مقدمه

برنج دومين غله جهان است و غذاى اصلى بيش از نيمى

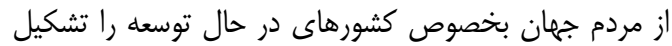

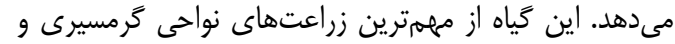

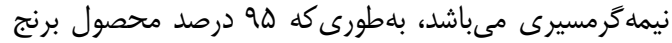

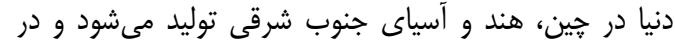

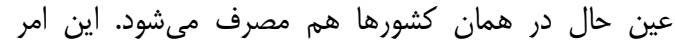

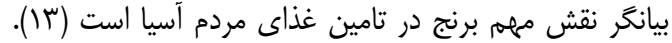

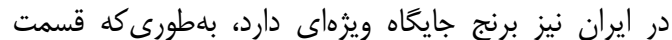

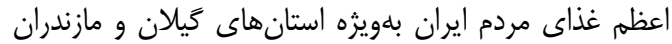

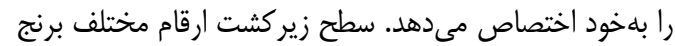

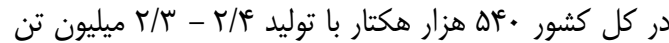

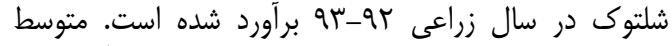

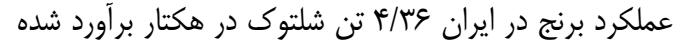

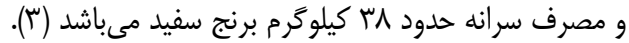

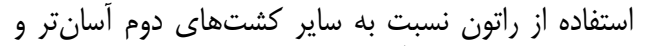

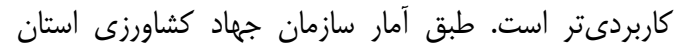

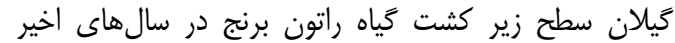

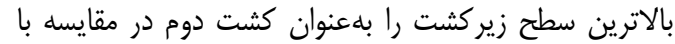

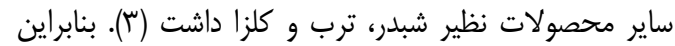

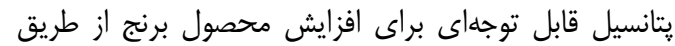

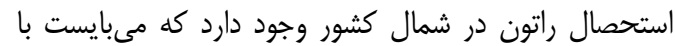

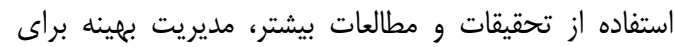

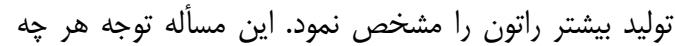


استخراج DNA از نمونههاى بركى با روش مورى موراى و

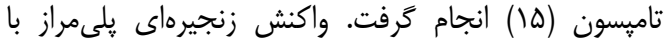

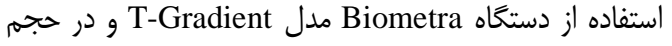

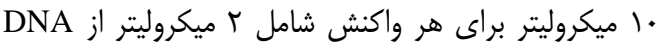

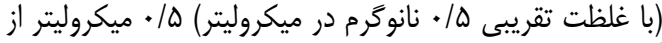

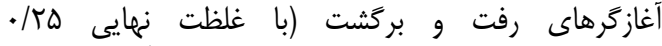

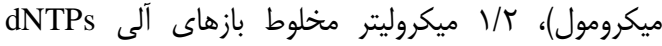

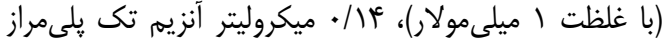

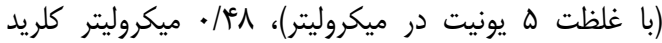

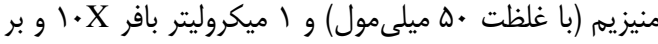

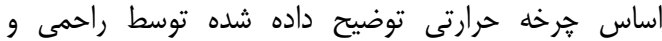

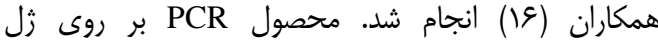

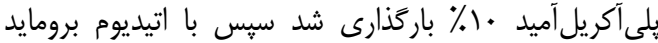

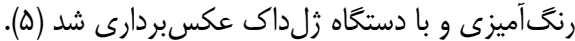

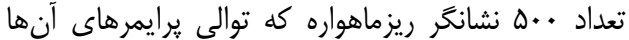

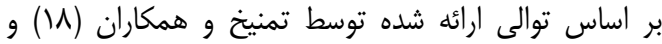

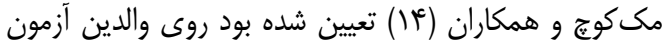

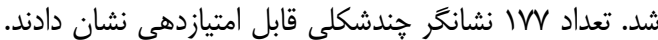

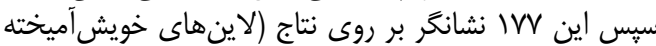

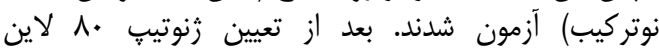

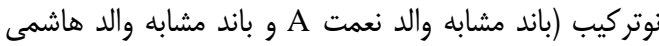

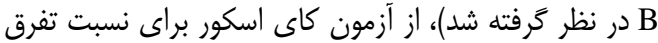

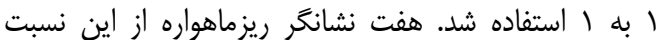

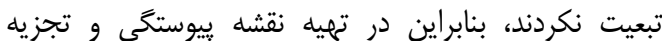

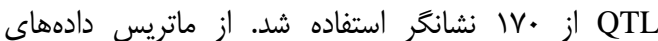

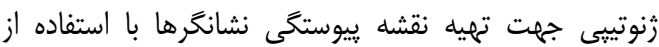
نرم|فزار 3.0

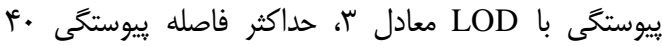

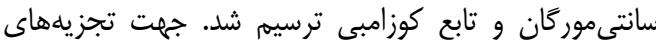

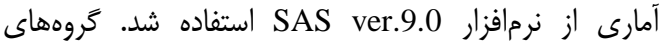

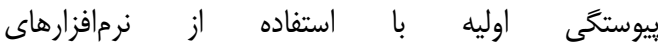
QTLIciMapping و (IT) MAPMAKER/EXP 3.0

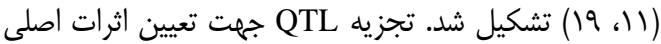

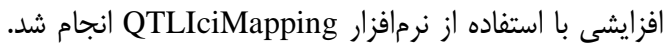

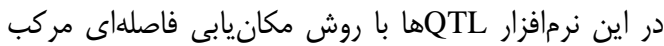

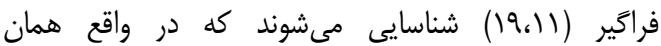

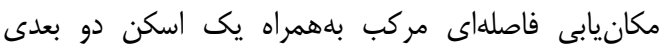

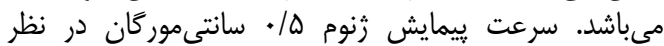

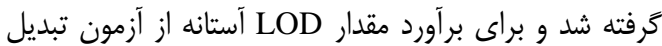

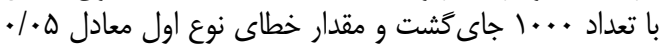

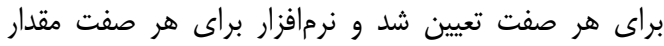

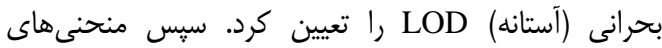

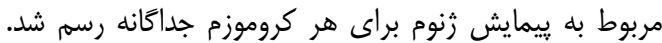

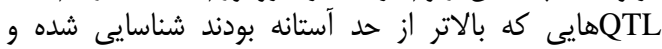

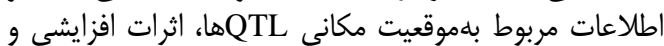
ميزان واريانس فنوتيبى (درصد) آنها توسط نرمافيت افزار مشخص أنسان
تاقى بركشتى بين دو رقم نييونباره (Nipponbare) و و

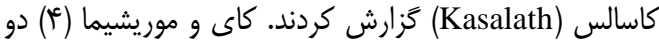

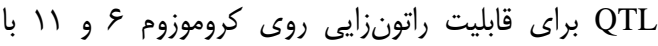

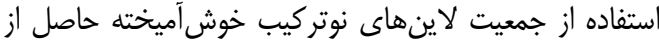
تلاقى بين Oryza rufipogon و يكى ريك رقم اينديكا به نام

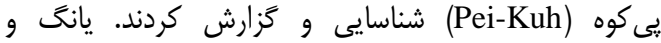

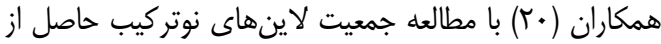

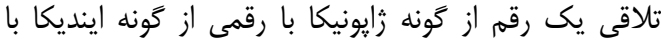

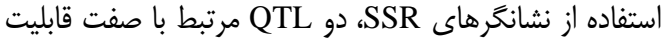
راتونزايى روى كروموزومهاى أ و ه ها را شناسايى كردندا جنى

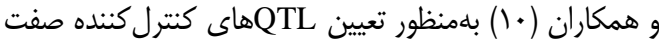

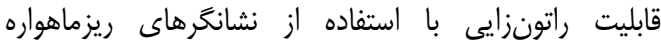

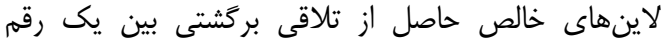

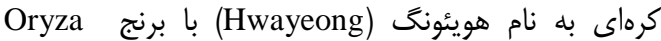
QTL rufipogon

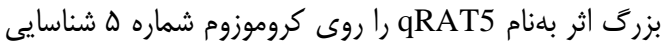

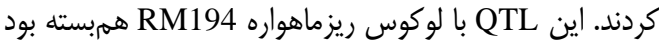

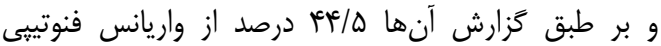

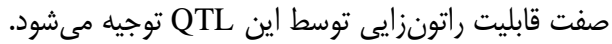

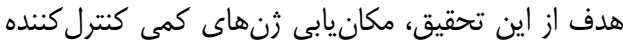

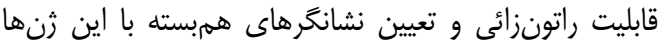

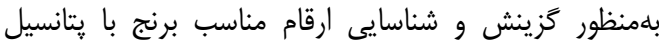

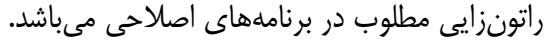

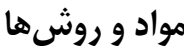

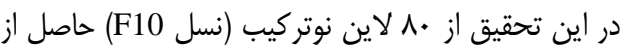

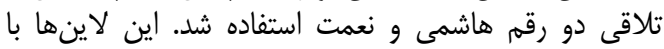

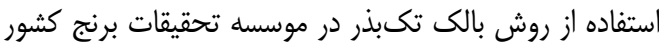

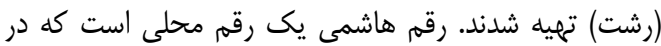

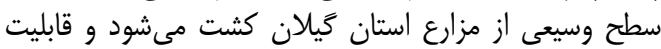

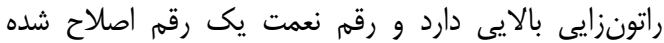

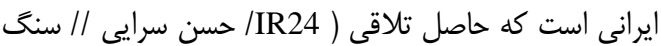

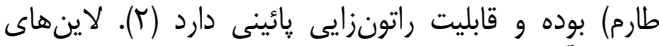

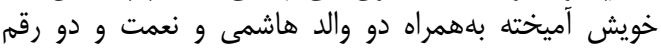

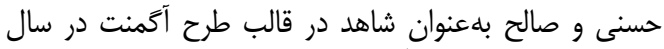

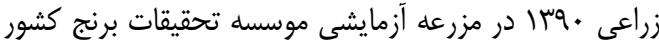

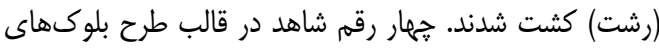

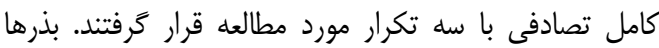

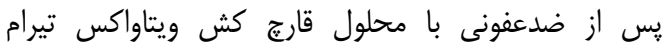

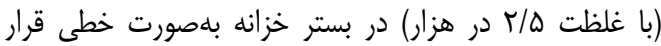

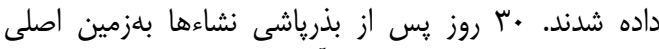

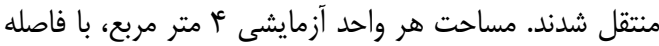

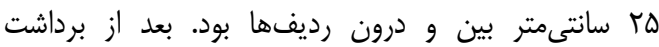

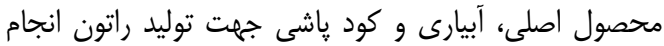

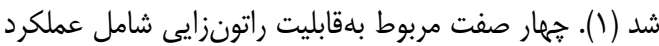

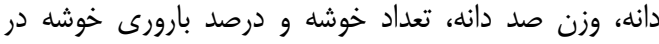
محصول راتون لاينها و ارقام شاهد اندازمخيرى شدند. 


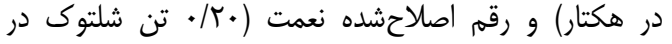

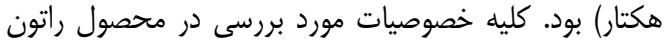

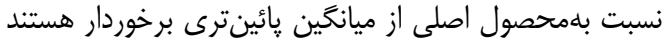

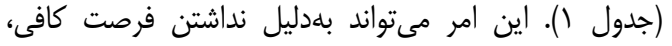

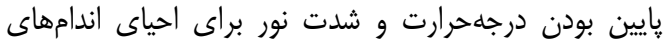

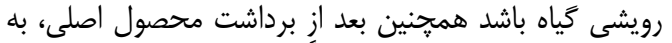

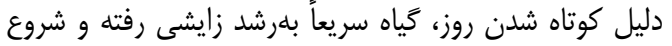

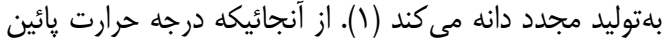

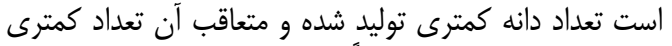

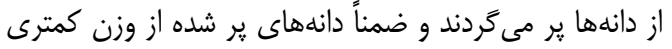

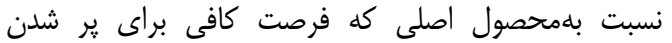

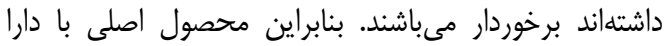

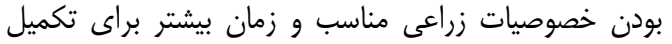

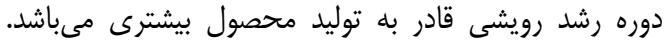

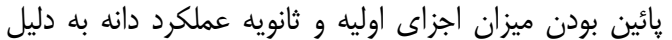

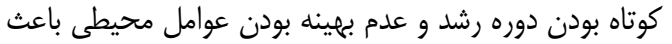

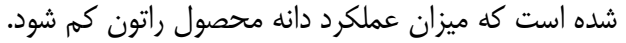

\section{نتايج و بحث تجزيه آمارى دادههاى فنوتيبى نتائ}

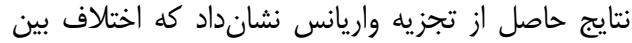

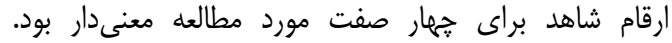

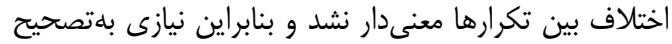

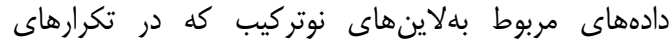

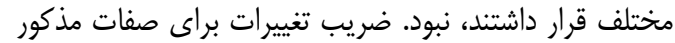

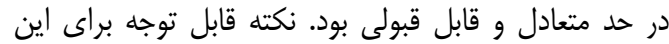

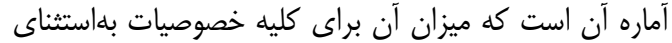

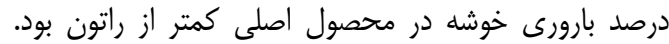

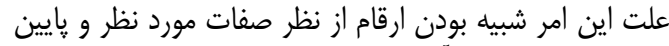

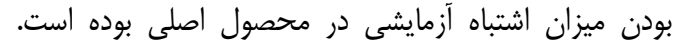

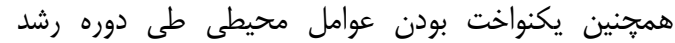
محصول اصلى مىتواند يكى از عوامل كاهش اشتي اشتباه آزمايشى

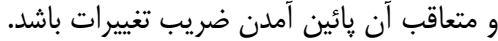

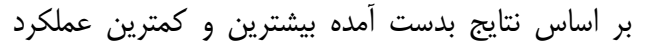

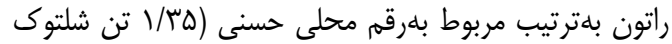

جدول ا- ميانگين خصوصيات مهم زراعى (اصلى و راتون) در ارقام شاهد

Table 1. Means of important agronomical traits of ratooning and main crop in control varieties

\begin{tabular}{|c|c|c|c|c|c|c|c|c|c|}
\hline \multicolumn{2}{|c|}{ 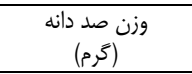 } & \multicolumn{2}{|c|}{$\begin{array}{c}\text { تعداد خوشه } \\
\text { تعدد) }\end{array}$} & \multicolumn{2}{|c|}{$\begin{array}{c}\text { بارورى خدرشه } \\
\text { (درصد }\end{array}$} & \multicolumn{2}{|c|}{$\begin{array}{c}\text { (تن دمر هكتار) } \\
\text { (تكانه } \\
\end{array}$} & \multirow[t]{2}{*}{ ارقام } & \multirow[t]{2}{*}{ رديف } \\
\hline راتون & اصلى & راتون & اصلى & راتون & اصلى & راتون & اصلى & & \\
\hline $1 / \Delta$ & $\mu / \Lambda$ & $N / V$ & $1 f / 9$ & V. & $M / G$ & $T / \tau$. & $r / \Delta V$ & هاشمى & 1 \\
\hline $1 / \pi$ & $\Gamma / \Delta$ & $\Delta / \Gamma$ & $1 \mathrm{~V} / \mathrm{q}$ & $8 \cdot / \mathrm{V}$ & $\Lambda \mathcal{N} / \mathcal{F}$ &.$\pi$. & $8 / 94$ & نعمت & $r$ \\
\hline $1 / 4$ & $\Gamma / \Lambda$ & $9 / \pi$ & $\mid f / 1$ & $n$ & $91 / 8$ & מז/ו & $r / 4$. & حسنى & r \\
\hline $1 / 4$ & $r / 1$ & $\mathrm{~V}$ & $10 / \Gamma$ & $9 \Delta$ & AV & מז/. & $\Gamma / \Delta \Delta$ & صالح & f \\
\hline $1 / 4$. & T/ץ & $\mathrm{V} / \Delta \Lambda$ & $1 \Delta / \Delta \omega$ & 89191 & $M / F$. & $\cdot / \mathrm{VA}$ & $F / \Delta$. & & \\
\hline.$/ .1$ &.$/ K Y$ & $1 / \Lambda$. & $1 / 94$ & $r / V V$ & $T / \mu r$ & $\cdot / \Delta \mathrm{A}$ & $1 / 41$ & & \\
\hline
\end{tabular}

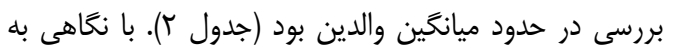

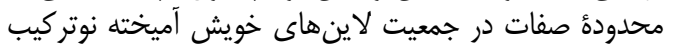

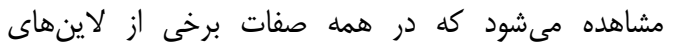

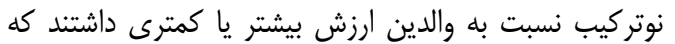

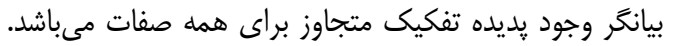

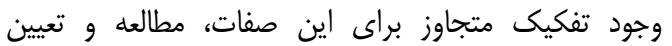

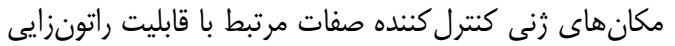

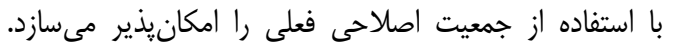

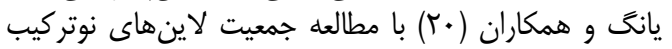

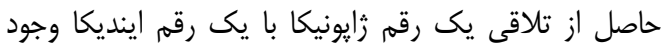

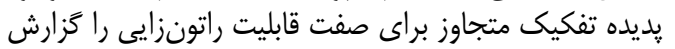
نمودند.
ميانكين و اشتباه استاندارد والدين و لاينهاى خوريش بردي

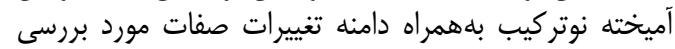

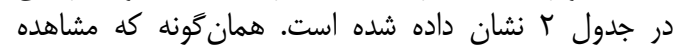

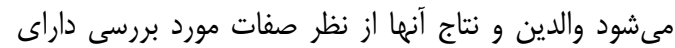

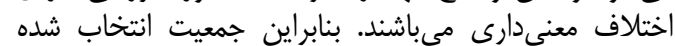

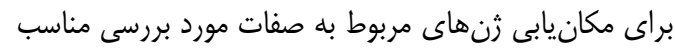

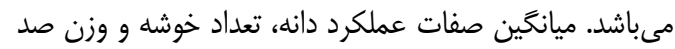

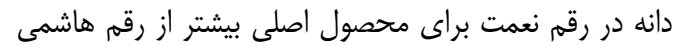

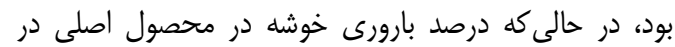

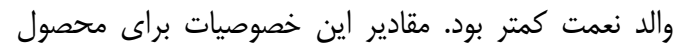

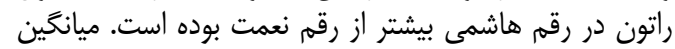
لاينهاى خويش آميخته نوتركيب، در تمامى صفات مورد مورد

جدول r- ميانگين، اشتباه استاندارد و دامنه تغييرات صفات در والدين و لاينهاى خويش آميخته نوتر كيب Table 2. Means, standard error and variation range of traits in parents and recombinants inbred lines (RILs)

\begin{tabular}{|c|c|c|c|c|c|c|c|}
\hline \multicolumn{4}{|c|}{ جمعيت لاين هاى خويش آميخته نوتر كيب } & \multicolumn{2}{|c|}{ والدين (اشتباه استاندارد土 ميانكين) } & \multirow{2}{*}{\multicolumn{2}{|c|}{ صفت }} \\
\hline دامنه & حداكثر & حداقل & اشتباهاستانداردد ميانكين & نعمت & هاشمى & & \\
\hline r/T & $\Delta / 8 \Delta$ & שT/T & T/RGD./TGT & $9 / r K \pm \cdot / \cdot q$. & $r / \Delta V \pm \cdot / \mu F$ & اصلى & عملكرد دانه \\
\hline $1 / r$. & $1 / \pi \omega$ & .110 & $\cdot / q \wedge \pm \cdot / \cdot r q$ & $\cdot / r \cdot \pm \cdot / \cdot \cdot t$ & T/4. & راتون & (تن در هكتار) \\
\hline$|V / r|$ & $9 / / V$ & $V \Delta / \Delta$. & $V N / I \cdot \pm \cdot / \Delta T \cdot$ & $\Lambda \& / F \cdot \pm \cdot / r \cdot V$ & $M / \varepsilon_{ \pm} \cdot / T V \Lambda$ & اصلى & بارورى خوشه \\
\hline$r F / v$. & $\Lambda \Delta / \Delta \omega$ & $\Delta \cdot / \Lambda \Delta$ & $G N / r \cdot \pm \cdot / F \Delta D$ & $G \cdot / V \cdot \pm \cdot / T \Delta \mid$ & $V \cdot / \cdot \cdot \pm \cdot / F \Delta q$ & راتون & (درصد) (د) \\
\hline १/.. & $r / \omega$. & $I T / \Delta$. & $\left.19 / \pi \omega_{ \pm} \cdot / 9 q\right)^{f}$ & $I V / q \cdot \pm \cdot / r q 4$ & $\mid \varphi / 9 \cdot \pm \cdot / \mu \omega$ & اصلى & ت تعداد خوشه \\
\hline $8 / 10$ & $1 . / \% \omega$ & $F / r$. & $\checkmark / Q \Lambda \pm \cdot / \% \Delta \Delta$ & $\Delta / r \cdot \pm \cdot / T \cdot \cdot$ & $N / V \cdot \pm \cdot / T+1)$ & راتون & (عدد) \\
\hline.$/ 9$. & $r / \& \Delta$ & $r / \bullet$ & $T / T_{ \pm \pm} \cdot / \cdot F_{0}$ & $\Gamma / \Delta \cdot \pm \cdot /$ YQD & r Tr// & اصلى & وزن صد دانه \\
\hline$\cdot / \mathrm{N}$ & I/Va & $1 / .4$ & $1 / 4 \cdot \pm \cdot / \cdot r \Delta$ & $1 / 4 \cdot \pm \cdot 1 \cdot 40$ & $1 / \Delta \cdot \pm \cdot / \cdot \Delta \Lambda$ & راتون & (َرم) \\
\hline
\end{tabular}


از نظر خصاصوصيات در محصول اصلى و راتون متفاوت مىباشد

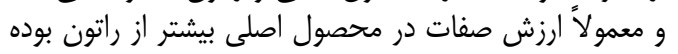

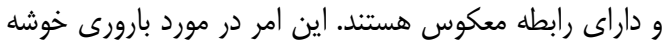

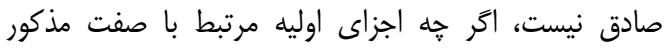

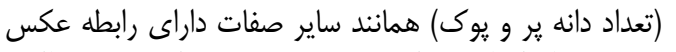

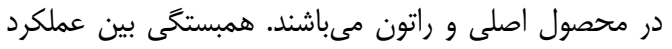

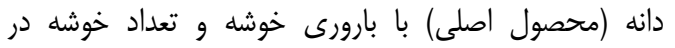

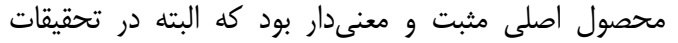

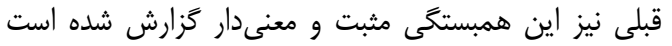

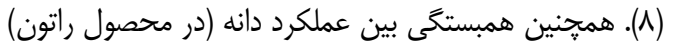

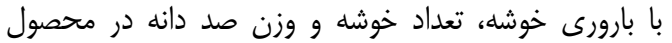
راتون نيز مثبت و معنىدار بود.

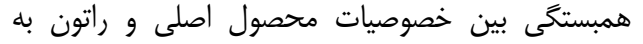

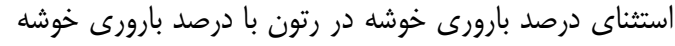

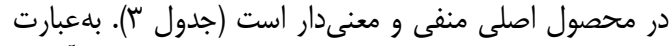

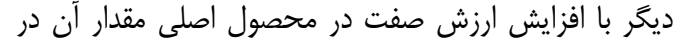

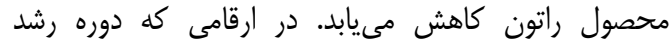

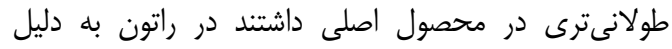

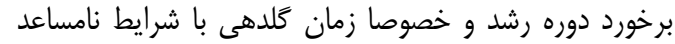

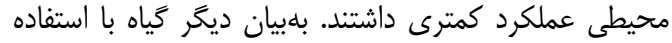

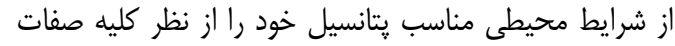

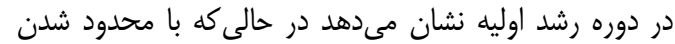

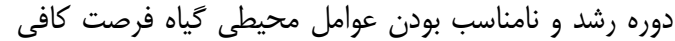

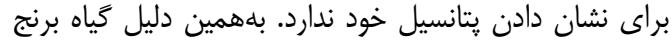

جدول س- ضرايب همبستكى ييرسيون بين صفات مرتبط با قابليت راتونزايى در جمعيت لاينهاى خويش آميخته نوتر كيب Table 3. Correlation coefficients between of ratooning ability traits in RILs population

\begin{tabular}{|c|c|c|c|c|c|c|c|c|c|}
\hline & & \multicolumn{2}{|c|}{ عملكرد دانه } & \multicolumn{2}{|c|}{ بارورى خوشه } & \multicolumn{2}{|c|}{ تعداد خوشه } & \multicolumn{2}{|c|}{ وزن صد دانه } \\
\hline & & اصلى & راتون & اصلى & راتون & اصلى & راتون & اصلى & راتون \\
\hline \multirow{2}{*}{ عملكرد دانه } & اصلى & 1 & - & & & & & & \\
\hline & راتون & $-\cdot / M \Lambda \Delta^{T \pi}$ & 1 & & & & & & \\
\hline \multirow{2}{*}{ بارورى خوشه } & اصلى & $\cdot / \mathrm{V} \wedge \Delta^{m \pi}$ &.$/ 9.1^{-\pi}$ & 1 & & & & & \\
\hline & راتون &.$- / 9 V^{2-m}$ & ./q\& & $\cdot / \Lambda \& D^{T \pi}$ & 1 & & & & \\
\hline \multirow{2}{*}{ تعداد خوشه } & اصلى & $\cdot / 91 \cdot{ }^{\pi m}$ &.$- / 11 \Lambda^{\pi \tau}$ & $-\cdot / \mathrm{V} 9 \Delta^{\mathrm{T} T}$ & $-\cdot / 9 r v^{m \pi}$ & 1 & & & \\
\hline & راتون & $-\cdot / 9 \vee \Lambda^{\pi-\pi}$ &.$/ 9 \Delta V^{* \pi}$ & $\cdot / M r^{T T}$ &.$/ 999^{\circ-\pi}$ &.$- / 9 \uparrow q^{n \pi}$ & 1 & & \\
\hline \multirow[b]{2}{*}{ وزن صد دانه } & اصلى & $\cdot / \cdot 1 f^{\mathrm{ns}}$ & $\cdot / r \wedge 9^{n s}$ & $\cdot 18 \cdot 9^{n \pi}$ & . & $-\cdot 1 \cdot+\omega^{\mathrm{ns}}$ &.$/ A q r^{n s}$ & 1 & \\
\hline & راتون & $-\cdot / \Lambda F \cdot{ }^{\pi T}$ & $.1899^{-10}$ & $\cdot / r \wedge \varepsilon^{n s}$ & $\cdot / V 99^{m-x}$ & $-\cdot / V F \theta^{\pi T}$ & $\cdot / \mathrm{V} \cdot{ }^{\pi+r}$ & $-\cdot / 4 \lambda .^{*}$ & 1 \\
\hline
\end{tabular}

ميانگين صفات مورد بررسى در شكل ا نشان داده شده است.

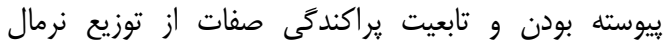

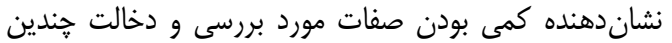

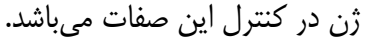

توزيع فراوانى لاينهاى جمعيت لاينهاى خويش آميخته

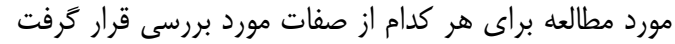

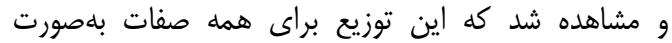

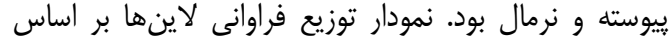
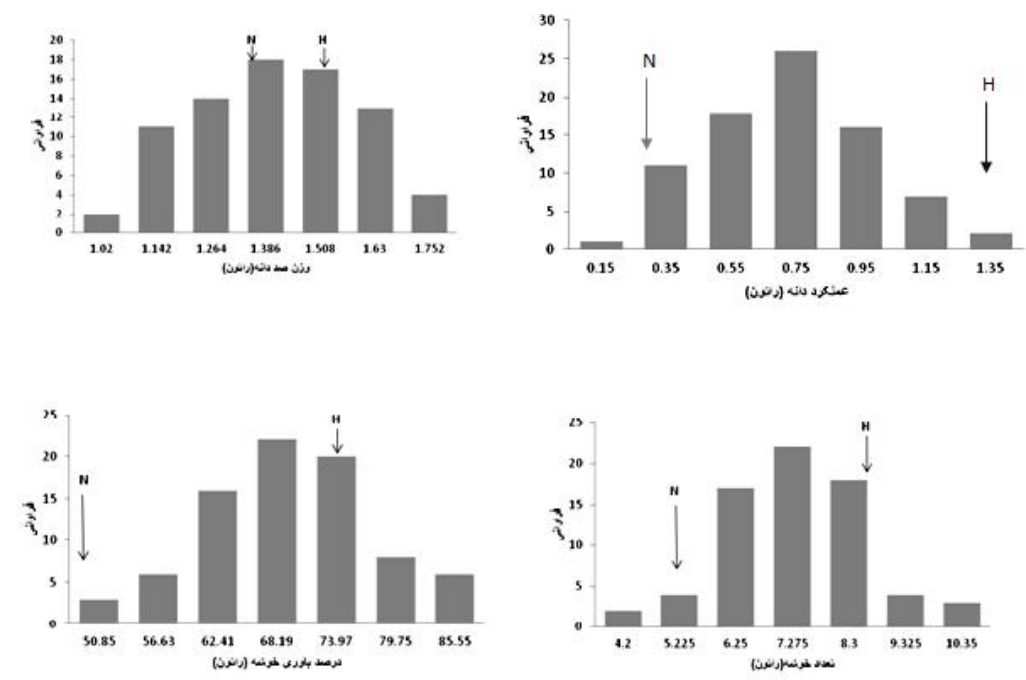

شكل ا- توزيع فراوانى لاينهاى خويش آميخته نوتر كيب براى صفات مرتبط با قابليت راتونزايى

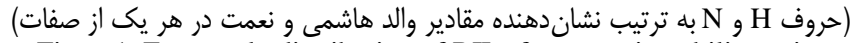

Figure1. Frequently distribution of RILs for ratooning ability traits

(H and $\mathrm{N}$ Letters showed traits value in Hashemi and Neamat) 


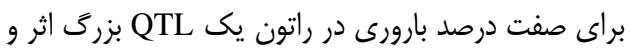

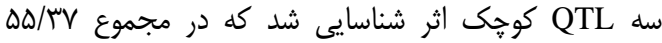

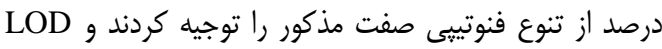

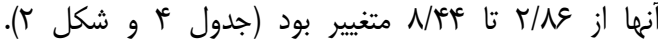

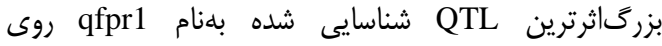
كروموزوم 1 و و دراترترين

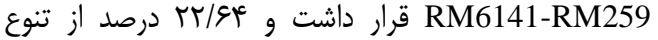
فنوتييى را براى اين صفت توجيه كرد و دارایى اثر افزايشى داري

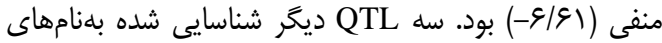

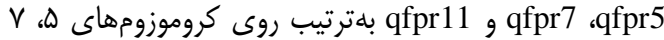
و ال القرار داشتند.

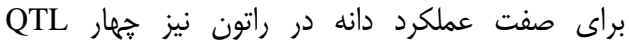

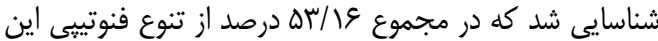

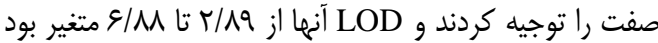

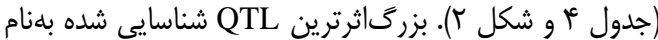

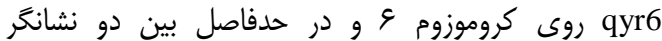
فرتئ RM4128-RM402

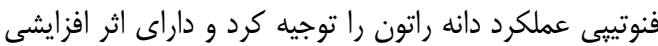

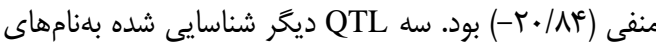

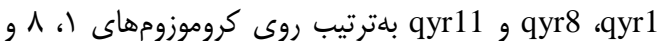

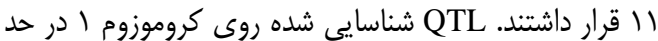

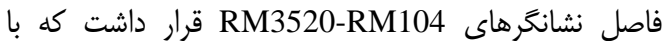

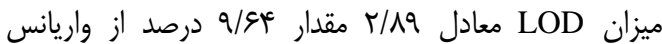

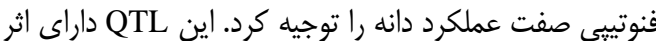

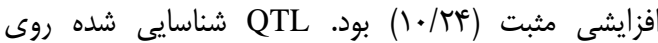

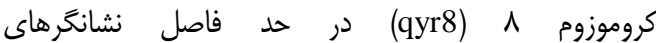

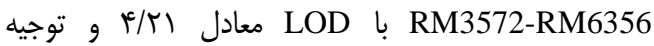
IT/V.

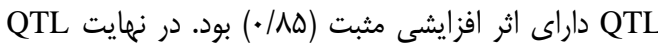

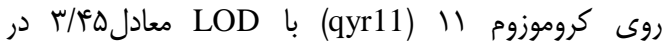

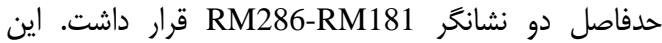

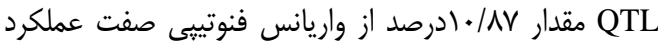

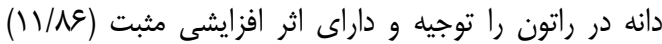

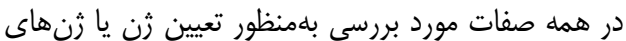

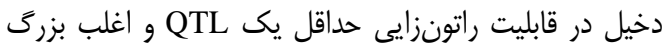
اثر روى كروموزوم 1 شناسايى شد شدافي كه البته

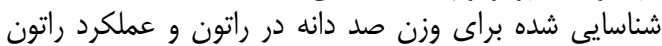

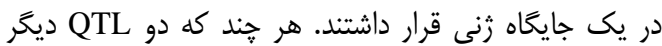

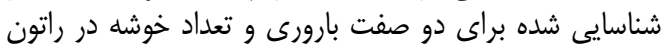

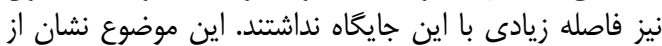

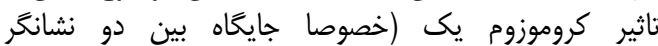

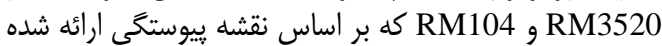

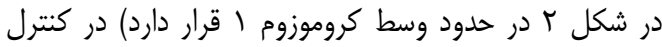

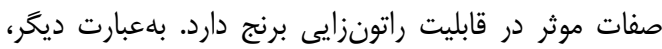

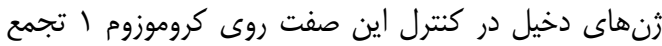

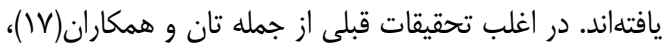

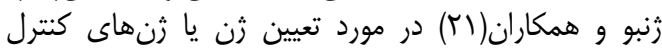

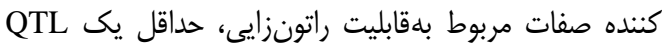

تهيه نقشه بيوستخى نشانكر ها

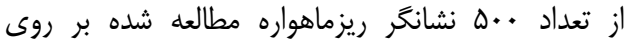

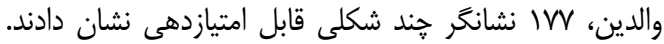

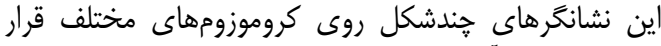

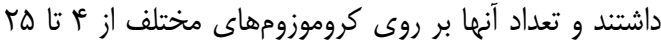

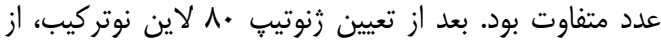

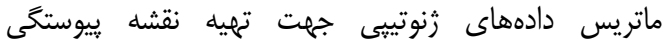

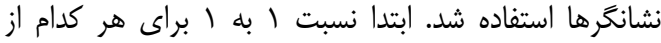

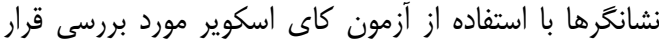

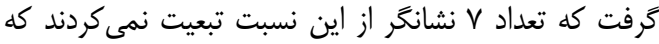

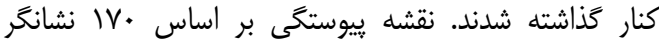

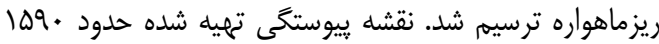

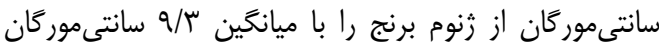

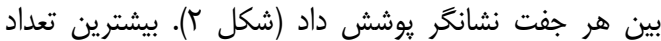

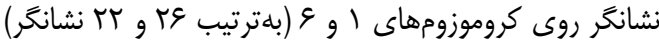

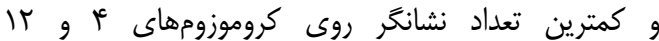

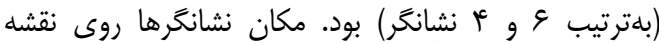

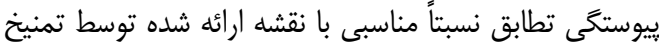

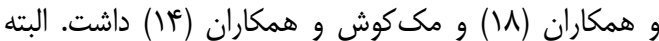

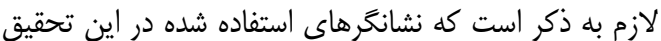

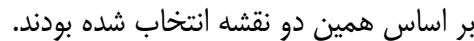
مكانيابى QTL هاى كنترل كنتده صفات

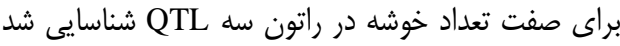

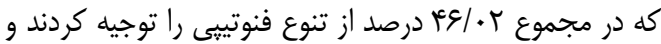

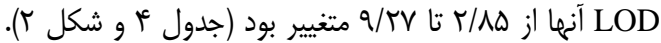
بزرى اثرترين QTL شناسايى شده بلنام

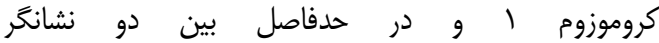
قرار داشت و r RM265-RM302

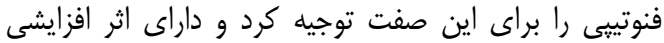

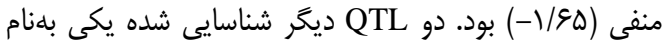
qpfr3

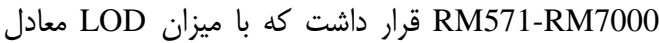

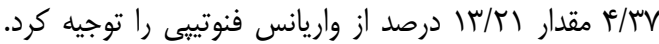

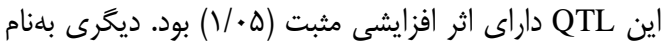
qpfr5 RM6645-RM163

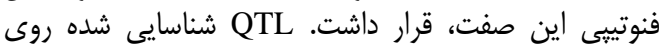

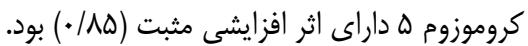

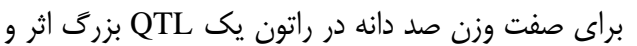

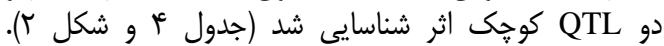
بزرگاثرترين QTL شناسايى شده بلنام

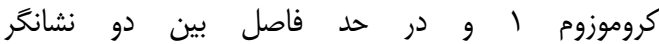

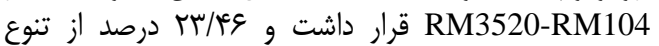

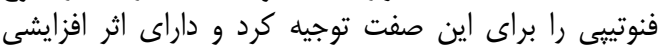

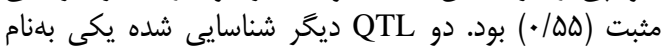

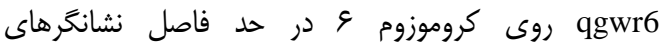

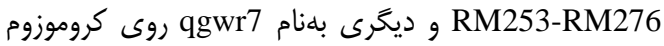
V در حداصل نشانكرهاى RM8263-RM8006 قرار داشتند. 
دانه در خوشه، توسط محققين قبلى شناسايى و معرفى شده

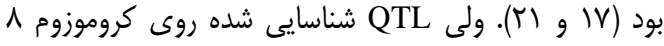

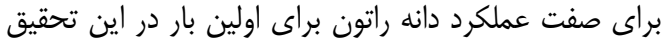

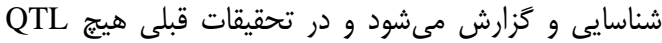

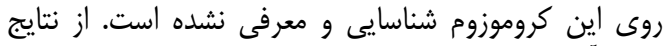

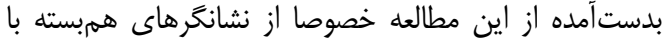

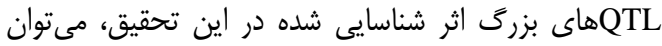

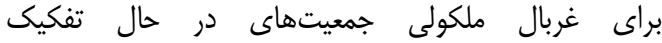

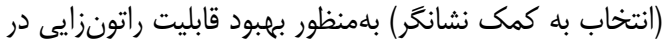
ارقام مختلف برنج استفاده نمود.

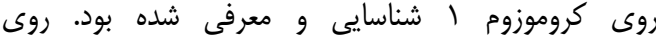

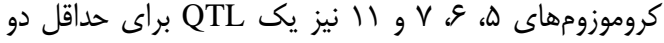

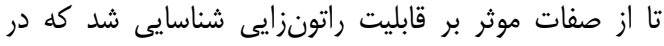

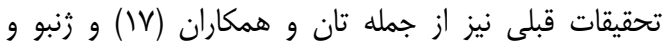

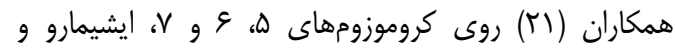

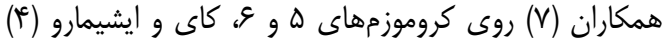

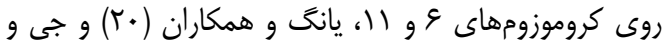

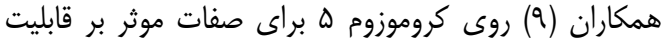

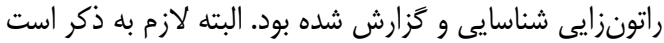

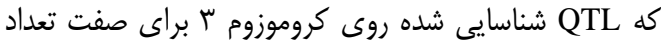
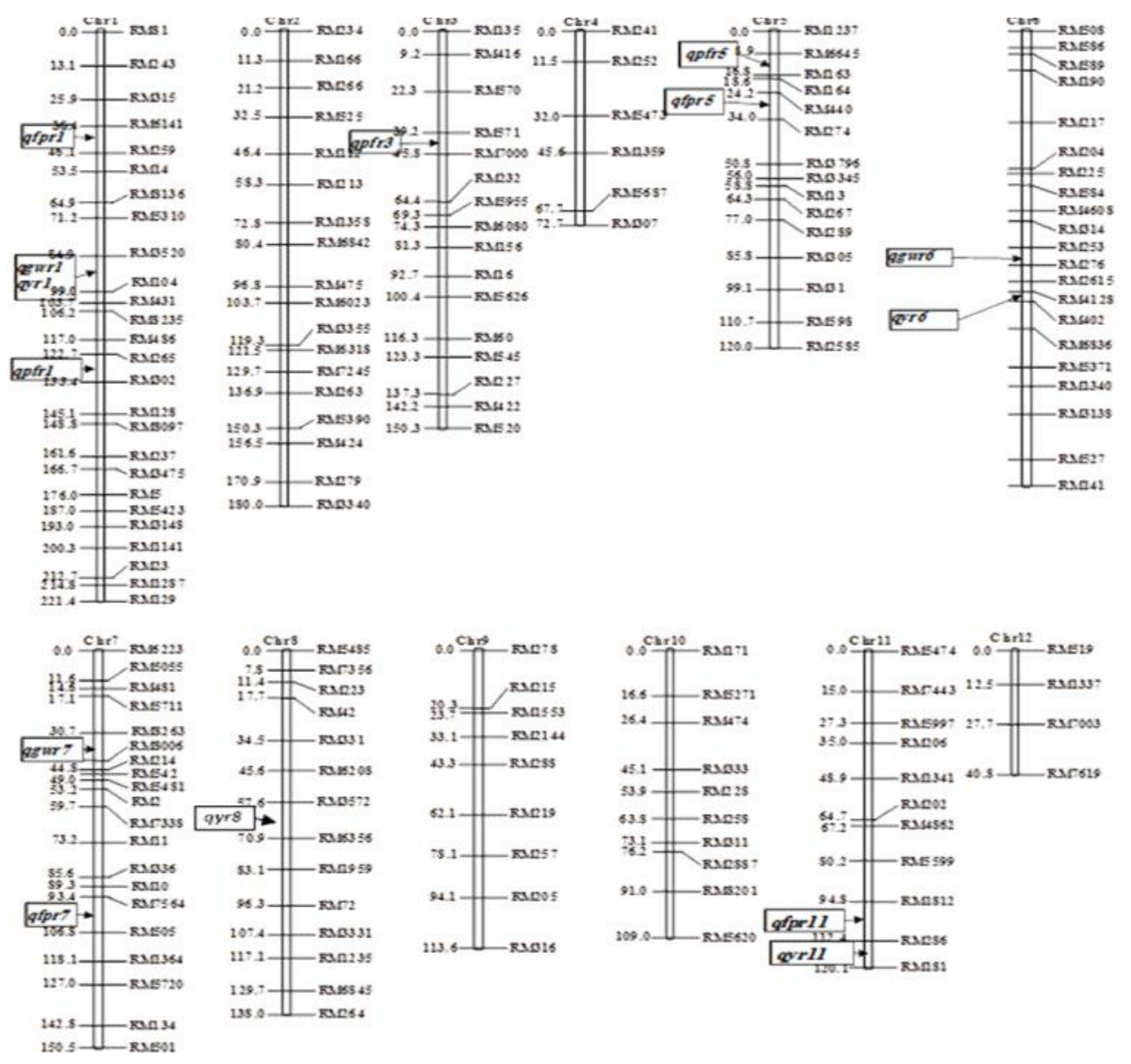

شكل r- نقشه لينكازى نشانگرهاى ريزماهواره روى كروموزومهاى برنج به همراه QTL شاى شناسايى شده براى صفات

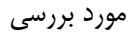

Figure 2. SSR linkage maps of rice chromosomes with identified QTLs for studied traits 
RILs هاى شناسايى شده بر اساس روش مكانيابى فاصلهاى مركب فراكير براى صفات قابليت راتونزايى برنج در جمعيت QTL Table 4. Identified QTLs based on Inclusive composite interval mapping method for ratooning ability traits in RILs

\begin{tabular}{|c|c|c|c|c|c|c|c|}
\hline درصد واريانس & اثر افزايشى & LOD & LOD آستانه & نشانكر & كروموزوم & QTL & صفات \\
\hline$|r / T|$ & $1 / \cdot \Delta$ & $F / r V$ & $r / \Lambda$ & RM571-RM7000 & $r$ & qpfr3 & \multirow{3}{*}{ نعداد خوشه در راتون } \\
\hline$V / \& 9$ & $\cdot / \Lambda \Delta$ & $r / \Lambda \Delta$ & & RM6645-RM163 & $\Delta$ & qpfr5 & \\
\hline$T \Delta / T^{2}$ & $-1 / 90$ & $Q / T V$ & & RM265-RM302 & 1 & qpfr1 & \\
\hline$r r / \& \Lambda$ & $\cdot / \Delta \Delta$ & N/Q9 & $r / \Gamma$ & RM3520-RM104 & 1 & qgwr1 & \multirow{3}{*}{ وزن صد دانه در راتون } \\
\hline $\mid F /$ & $-/ T \omega$ & $\Delta / \Gamma 1$ & & RM253-RM276 & \& & qgwr6 & \\
\hline$I T / V F$ &.$- / \mu \cdot$ & $r / v r$ & & RM8263-RM8006 & v & qgwr7 & \\
\hline$T r / a t$ & $-9 / 9 \mid$ & $N / F^{4}$ & $r / v \Lambda$ & RM6141-RM259 & 1 & qfpr1 & \multirow{4}{*}{ بارورى خوشه در راتون } \\
\hline $\mid r / 90$ & $-r / 11$ & $F / \Delta q$ & & RM440-RM274 & $\Delta$ & qfpr5 & \\
\hline $1 \cdot / 4$ & r/gr & r/T & & RM7564-RM505 & $v$ & qfpr7 & \\
\hline ৭/^১ & T/AT & $r / \mathcal{L}$ & & RM1812-RM286 & 11 & qfpr11 & \\
\hline $19 / 90$ & $-T / A F$ & g/M & $r / 9 \Lambda$ & RM4128-RM402 & 9 & qyr6 & \multirow{4}{*}{ عملكرد دانه در راتون } \\
\hline $9 / 94$ & $1 \cdot / K F$ & $r / 19$ & & RM3520-RM104 & 1 & qyr1 & \\
\hline $\mathrm{IT} / \mathrm{V}$ & | & $r / r)$ & & RM3572-RM6356 & $\wedge$ & qyr8 & \\
\hline $1 . / A V$ & $\Pi / \Lambda \varepsilon$ & $r / \mp \Delta$ & & RM286-RM181 & 11 & qyr 11 & \\
\hline
\end{tabular}

مىدانند از سازمان تحقيقات، آموزش و ترويج كشاورزى و

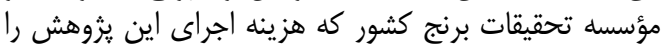
تقبل نمودهاند، صميمانه تشكر نمايند.

\author{
تشكر و قدردانى

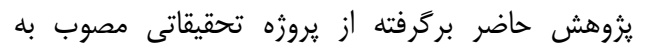

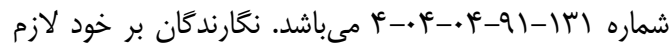

1. Akhgari, H. 1996. Determination of ratoon yield potential in rice (Oryza sativa L.) cultivars. M.Sc Thesis, Islamic Azad University, Karaj Branch, Iran (In Persian).

2. Allahgholipour, M., A.J. Ali, F. Alinia, T. Nagamine and Y. Kojima. 2006. Relationship between rice grain amylose and pasting properties for breeding better quality rice varieties. Plant Breeding, 125: 357-362.

3. Anonymouse. 2015. Agricultural statistics, the first volume agricultural crops 2013-2014. Ministry of Agriculture, Department of Planning and Economy, Office of Statistics and Information Technology, (In Persian).

4. Cai, H.W. and H. Morishima. 2002. QTL clusters reflect character associations in wild and cultivated rice. Theoretical and Applied Genetics, 104: 1217-1228.

5. Ebadi, A. A., E. Farshadfar and B. Rabiei. 2013. Mapping QTLs controlling cooking and eating quality indicators of Iranian rice using RILs across three years. Australian Journal of Crop Science, 10: 1494-1502.

6. Hashemi Dezfoli, A., A. Kochaki and M. Benayan Avval. 1995. Increasing crop yield. Mashhad Jahad Daneshgahi Press. First edition, 287 pp (In Persian).

7. Ishimaru, K., M. Yano, N. Aoki, K. Ono, T. Hirose, S. Y. Lin, L. Monna, T. Sasaki and R. Ohsugi. 2001. toward the mapping of physiological and agronomic characters on a rice function map: QTL analysis and comparison between QTLs and expressed sequence tags. Theoretical and Applied Genetics, 102: 793-800.

8. Jahani M., G. Nematzadeh and G. Mohammadi Nejad. 2016. Evaluation of Agronomic Traits Associated with Grain Yield in Rice (Oryza sativa) Using Regression and Path Analysis. Journal of Crop Breeding. 7: 115-122 (in Persian).

9. Ji, S., X. Luo and S.N. Ahn. 2014. Mapping QTL for ratooning ability in advanced backcross lines from an Oryza sativa x O. rufipogon cross. CNU Journal of Agricultural Science, 41: 1-7.

10. Karbalaei, M., N. Sharafi, R. Erfani and Gh. Nematzadeh. 1997. The potential for increased rice production as a function ratoon and studies. The publication Rice Research Institute. First edition. 55 pp (In Persian).

11. Li, H., J.M. Ribaut, Z. Li and J. Wang. 2008. Inclusive composite interval mapping (ICIM) for digenic epistasis of quantitative traits in biparental populations. Theoretical and Applied Genetics, 116: 243-260.

12. Lincoln, S. E., M.J. Daly and E.S. Lander. 1990. Constructing genetic linkage maps with MAPMAKER: a tutorial and reference manual. Technical Report. Cambridge, MA: Whitehead Institute for Biomedical Research.

13. Lu, B.R. and A. A. Snow. 2005. Gene flow from genetically modified rice and its environmental consequences. Biology Science, 55: 669-678.

14. McCouch, S.R., L. Teytelman, Y.B. Xu, K.B. Lobos, K. Clare, M. Walton, B. Fu, R. Maghirang, Z.K. Li, Y.Z. Xing, Q.F. Zhang, I. Kono, M. Yano, R. Fjellstrom, G. DeClerck, D. Schneider, S. Cartinhour, D. Ware and L. Stein. 2002. Development and mapping of 2240 new SSR markers for rice (Oryza sativa L.) DNA Research. 9: 199-207.

15. Murray, M.G. and W.F. Thompson. 1980. Rapid isolation of high molecular weight plant. DNA Nucleic Acids Research, 8: 4321-4325.

16. Rahemi M. R., Kazemitabar S.K., A. Moumeni and A.A. Ebadi, 2009. Evaluation of Genetic Diversity by Using of Link Maker for Amylase Content of Some Iranian Local Rice Cultivars. Journal of Crop Breeding, 1:1-10 (in Persian).

17. Tan, Z.B., L.S. Shen, Z.L. Yuan, C.F. Lu, Y. Chen, K.D. Zhou and L.H. Zhu.1997. Identification of QTLs for ratooning ability and grain yield traits of rice and analysis of their genetic effects. Acta Agronomica Sinica, 23: 289-295.

18. Temnykh, S., W.D. Park, N. Ayres, S. Cartinhour, N. Hauck, L. Lipovich, Y.G. Cho, T. Ishii, and S.R. McCouch. 2000 Mapping and genome organization of microsatellite sequences in rice (Oryza sativa L.) Theoretical and Applied Genetics, 100: 697-712.

19. Wang, J.K. 2009. Inclusive composite interval mapping of quantitative trait genes. Acta Agronomica Sinica, 35: 239245.

20. Yang, C.H., Y.P. Wang T.U. Bin, L.I. Ting, H.U. Liang and L.I. Shi-Gu. 2012. QTL analysis of rice Ratooning ability and related agronomic traits by using RILs Populations. Acta Agronomica Sinica, 7: 1240-1246.

21. Zhenbo, T., S. Lishuang, Y. Zuolian, L. Chaofu, C.Ying, Z. Kaida and Z. Lihuang. 1997. Identification of OTLs for rationing ability and grain yield traits of rice and analysis of their genetic effects. Acta Agronomica Sinica, 23: 289-295. 


\title{
Analysis of Quantitative Trait Loci for Ratooning Ability in Rils Population of Rice
}

\section{Ali Akbar Ebadi ${ }^{1}$, Mehrzad Allahgholipour ${ }^{2}$ and Naser Sharafi ${ }^{3}$}

\author{
1- Assistant Professor, Rice Research Institute of Iran, Agricultural Research Education and Extension \\ Organization (AREEO), Rasht, (Corresponding Author: ebady_al@yahoo.com) \\ 2 and 3- Assistant Professor and Instructor, Rice Research Institute of Iran, Agricultural Research Education and \\ Extension Organization (AREEO), Rasht \\ Received: November 30, $2015 \quad$ Accepted: September 25, 2016
}

\begin{abstract}
Tillering and secondary stems in graminea plants are permanent characters, whereas there were being food matters and proper temperature for regeneration. Ratooning or regeneration phase is explained to remain of plant for growing in next season. Recombinant inbred lines (RILs) consisting of 80 lines, derived from a cross between Hashemi and Nemat were used to analyze the genetic basis ratooning ability of rice. Recombinant inbred lines were tested with 177 polymorphic microsatellite markers. Linkage map were constructed with 170 microsatellite markers and its total lengths was $1590 \mathrm{cM}$ which the mean space between markers was $9.3 \mathrm{cM}$. Using of Inclusive composite interval mapping (ICIM) method, 14 QTLs were detected on chromosomes $1,3,5,6,7,8$ and 11 for the panicle number, hundred grain weight, fertility percentage and grain yield of ratoon. Among these mapped QTLs, qpfr1for panicle number, qgwr1 for hundred grain weight and qfpr1 for fertility percentage of ratoon , controlled $25.12 \%$, $23.46 \%$ and $22.64 \%$ of the phenotypic variance, respectively. The qyr6 was the major QTL for grain yield of ratoon on chromosome 6 , controlled $19.95 \%$ of the phenotypic variance. One new QTLs were identified for grain yield of ratoon which was located on chromosome 8.
\end{abstract}

Keywords: Rice, Ratooning ability, RILs, QTL mapping, SSR Markers 